\title{
An Updated Review of Dendrochronological Investigations in Mexico, a Megadiverse Country with a High Potential for Tree-Ring Sciences
}

\author{
Andrea C. Acosta-Hernández ${ }^{1}$, Marín Pompa-García ${ }^{1, *}$ and Jesús Julio Camarero ${ }^{2}$ \\ 1 Facultad de Ciencias Forestales, Universidad Juárez del Estado de Durango, Av. Papaloapan y Blvd. \\ Durango, Durango 34120, México; andrea_dgo@hotmail.com \\ 2 Instituto Pirenaico de Ecología (IPE-CSIC), Avda. Montañana 1005, 50059 Zaragoza, Spain; \\ jjcamarero@ipe.csic.es \\ * Correspondence: mpgarcia@ujed.mx; Tel.: +52-618-130-1096
}

Academic Editor: Glenn Juday

Received: 2 April 2017; Accepted: 5 May 2017; Published: 9 May 2017

\begin{abstract}
Dendrochronology is a very useful science to reconstruct the long-term responses of trees and other woody plants forming annual rings in response to their environment. The present review considered Mexico, a megadiverse country with a high potential for tree-ring sciences given its high climatic and environmental variability. We reviewed papers considering Mexican tree species that were published from 2001 to 2016. Most of these studies examined tree species from temperate forests, mainly in the pine and fir species. The review included 31 tree species. The most intensively sampled family and species were the Pinaceae and Douglas fir (Pseudotsuga menziessi (Mirb.) Franco), respectively. Some threatened tree species were also studied. Dendrochronological investigations were mainly conducted in northern and central Mexico, with Durango being the most sampled state. The reviewed studies were mostly developed for hydroclimatic reconstructions, which were mainly based on the tree-ring width as a proxy for the climate. Tree-ring studies were carried out in both national and foreign institutions. Our review identified relevant research gaps for dendrochronologists such as: (i) biomes which are still scarcely studied (e.g., tropical dry forests) and (ii) approaches still rarely applied to Mexican forests as dendroecology.
\end{abstract}

Keywords: conifers; dendroecology; dendroclimatology; tree rings

\section{Introduction}

Dendrochronology is the science studying annual growth rings in trees and other woody plants by relating characteristics of these rings with the environmental conditions in which they were formed [1]. The features of tree rings constitute an indirect source for understanding the historical characteristics of the environment in which trees have grown [2]. Usually, sensitive tree species are chosen because they form annual growth rings with certain amount of variability, which allows for synchronizing tree-ring series of different trees growing at the same site. In this way, the targeted tree species should be able to record different types of temporal signals in their rings due to the variability of environment conditions, especially the climate [3-5].

One of the first dendrochronological investigations was carried out in America by Andrew E. Douglass, who found a clear dependence between the width of growth rings in pine species from the southwestern part of United States of America (USA) and precipitation [6]. Other early contributions to tree-ring sciences in the neotropical regions were based on quantifying the activity of the vascular cambium as related to climate [7] or in the study of specific tree species, often considering their cambium phenology [8,9]. In the southern part of America, one of the first tree-ring studies 
examined Cedrela fissilis [10]. As the species and site conditions of the earliest dendrochronological studies developed in the southwestern part of USA are similar to those encountered in many northern Mexico forests, this promoted the theory that Douglass' school, including disciples such as E. Schulman, pioneered tree-ring studies in semi-arid northern American forests [11].

This research along with others stimulated the birth and development of dendrochronology, which promoted the realization of new studies mainly applied to reconstruct climate (dendroclimatology) [12]. Some of these studies are being developed in Mexico [13].

Mexico is considered one of the main Latin-American countries with a higher potential for dendrochronological investigations, due to its high environmental and climatic heterogeneity. These conditions result in a high diversity of gymnosperm (pines, firs and cypresses) or angiosperm tree species (oaks), which often show a high longevity [14-16]. The first dendrochronological studies of Mexico were carried out in places close to El Salto (Durango) city for some species of pines [11]. In the last decades, a large number of studies have been carried out, mainly focusing on dendroclimatology [17-19].

Although these studies are becoming more numerous in Mexico, we still do not know the state of the art in this scientific field. To our knowledge, there is no report that documents the current situation in the country of dendrochronology and the perspectives offered by dendrosciences. The systematization of this collection in a database would provide an overview to identify the background, knowledge gaps and trends that research has taken depending on the tree species, scientific scope, geographical region and type of ecosystem among other specific data. This constitutes a starting point for researchers interested in further developing dendrosciences in Mexico.

The objective of this study was to analyze the dendrochronological investigations that have been performed in Mexican terrestrial ecosystems, based on an exhaustive literature review to generate a diagnosis and synthesize the dendrochronological perspectives in this megadiverse country with a great tradition in forestry and ecology. In general, we expected to find more conifers and deciduous broadleaf tree species from seasonal climate zones in these studies. This is due to their growth rings being better defined, which facilitates their synchronization or cross-dating and subsequent measurement, compared with tropical forests in which tree-ring delimitation and cross-dating are not so simple $[1,20,21]$.

\section{Materials and Methods}

A bibliographic research of scientific articles developed in the subject of dendrochronology was done, using scientific Internet searcher engines (Web of Science-Thomson, Scopus, Science Direct, Google Academic, Redalyc). The research was performed considering studies published between the years of 2001 and 2016. The following English and Spanish keywords were used in: "dendrocronología", "dendrochronology", "anillos de crecimiento", "tree rings", "paleoclimatología", "paleoclimatology", "dendroclimatología", "dendroclimatology" "dendroecología" and "dendroecology". We included those publications based on studies that were developed in Mexico and excluded those that were not published in indexed journals, avoiding grey literature (thesis, memories of congresses, technical brochures, etc.).

From the research, a bibliographic database was built and analyzed, containing the following fields of information: vegetation type based on the classification of Land Use and Vegetation of the National Institute of Statistics and Geography (INEGI), studied tree species, site data (state, geographic coordinates and altitude), study objective and scope, journal where the study was published and measured variables (tree-ring width, earlywood and latewood widths, length of the series or chronologies) as well as the institution where the investigation was conducted. 


\section{Results}

A total of 55 articles were found in indexed journals (see the complete list in the Appendix A). These studies were carried out at altitudes ranging from 1300 to $4000 \mathrm{~m}$ above sea level (Figure 1). More than half of the studies (53\%) were carried out in forests located at more than $3000 \mathrm{~m}$ of elevation.

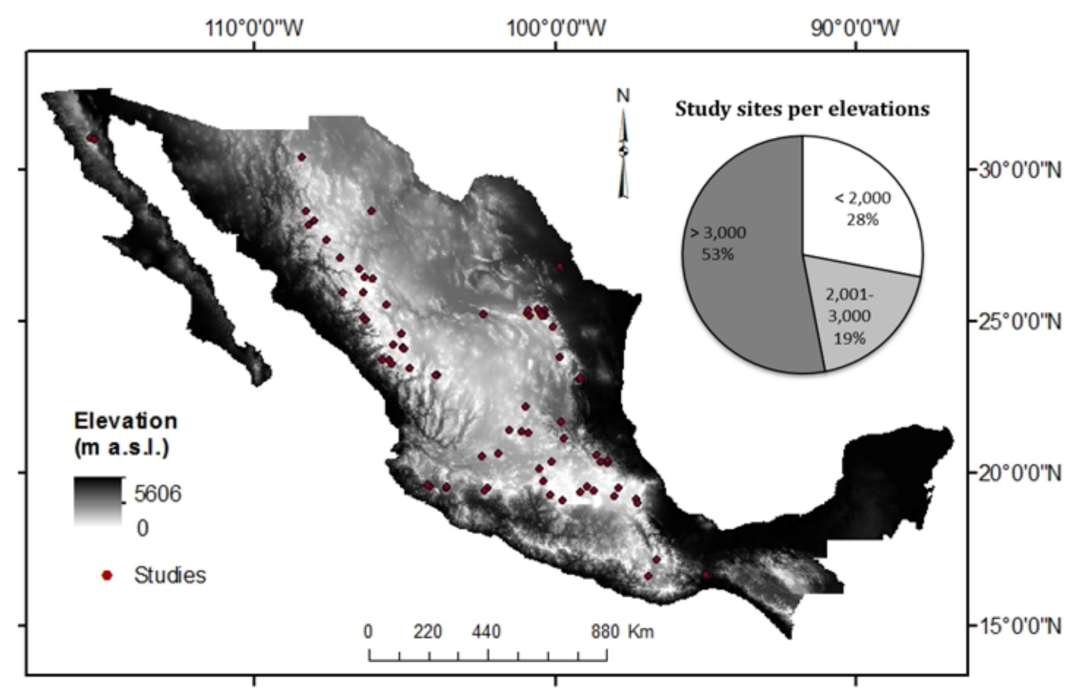

Figure 1. Sample sites of the dendrochronological studies carried out in Mexico. The upper right graph indicates the classification of sites based on their elevation.

Dendrochronological studies have been developed in seven different types of vegetation (Figure 2). Among them, $48 \%$ of the studies were developed for the pine species found within pine forests, which were the most studied type of vegetation, followed by the oyamel forest (Abies religiosa (Kunth) Schltdl. \& Cham.) in $24 \%$ of the studies. The pine-oak forest was found in $14 \%$ of the studies and the gallery forest accounted for $8 \%$. The types of vegetation that were less frequently studied included mountain mesophilous forest, coniferous scrubland and subtropical scrubland, with $2 \%$ of studies carried out in each one.

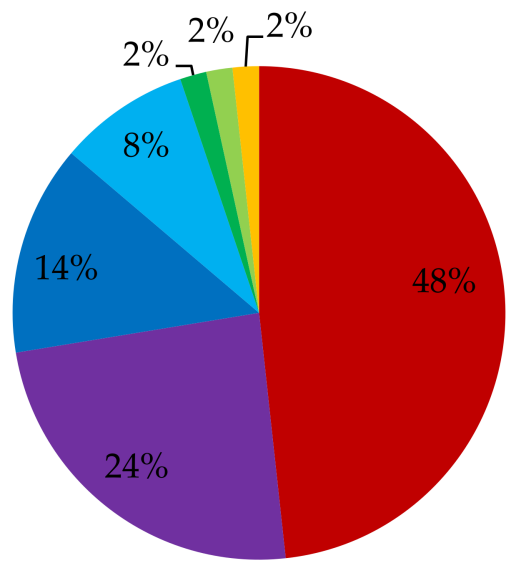

Pine forest

- Oyamel forest

Pine-oak forest

Gallery forest

Mountain

mesophilous forest

Figure 2. Percentages of studies classified according to the type of ecosystem following the classification of the National Institute of Statistics and Geography (INEGI).

Dendrochronological studies in Mexico examined 26 tree species, which were distributed in five families. The species of the Pinaceae family accounted for $82 \%$ of the studies, those of the Cupressaceae family accounted for $13 \%$. Finally, the families Oleaceae, Fabaceae and Fagaceae were only considered 
in $2 \%$ of the studies for each family (Figure 3). The most studied genera were Pinus, Taxodium, Pseudotsuga and Abies. Pinus was the most intensively studied (16 species), showing concordance with the great diversity of this genus present in Mexico that represents a world center in the diversity of pines [22].

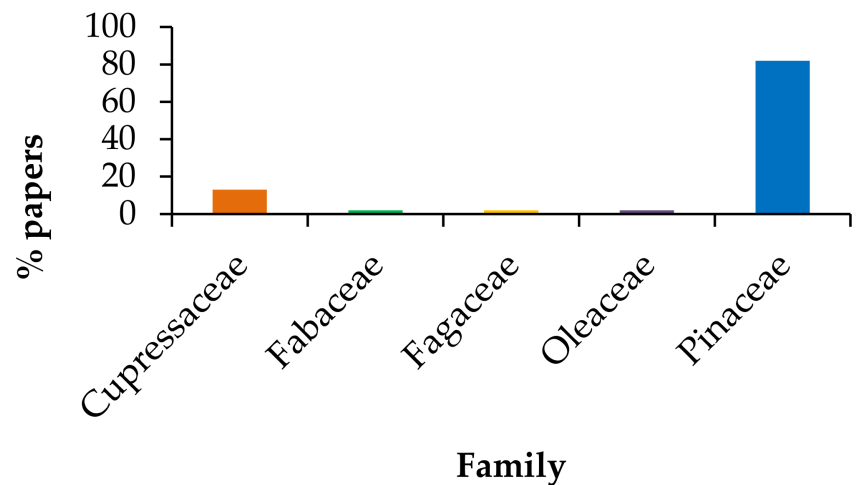

(a)

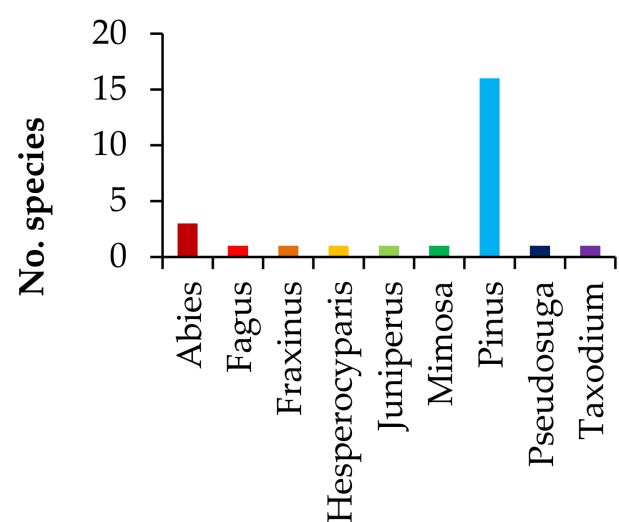

Genus

(b)

Figure 3. Dendrochronological studies performed in Mexico and grouped, according to (a) the taxonomic family and (b) the genus of the studied tree species.

The studied species and their frequency are reported in Figure 4 . The tree species with a greater number of studies is $P$. menziesii (16 studies), which is considered as one of the most important species in dendrochronology due to its climate sensitivity, good cross-dating and wide geographical distribution [23]. Another tree species frequently studied was Pinus cooperi C.E. Blanco (11 studies), which also has remarkable dendrochronological potential [24-27]. These two species were followed by Pinus hartwegii Lindl. and Taxodium mucronatum Ten. (5 studies).

There were six species studied that fall within a category of risk such as endangered $(\mathrm{P})$ or subject to special protection (Pr) according to the Official Mexican Standard 059 [28]: Abies concolor Lindl. (Pr), Juniperus monticola Martínez (Pr), Pinus jeffreyi Balf. (Pr), Pinus lagunae (Rob.-Pass.) Passini (Pr), P. menziesii (Pr) [29] and Pinus pinceana Gordon \& Glend. (P) (Figure 4). This section may be divided by subheadings. It should provide a concise and precise description of the experimental results, their interpretation as well as the experimental conclusions that can be drawn.

From the geographical point of view, dendrochronological studies in Mexico have been carried out in 20 states. Durango is the state most intensively sampled, followed by the states of Mexico, Chihuahua and Coahuila (Figure 5). These studies focused on the northern and central mountainous 
areas of the country, mostly in the Sierra Madre Occidental complex, which contains many temperate forests. A few studies in tropical ecosystems were found, whose research can be considered incipient in relation to other Latin-American countries $[16,21,30,31]$.

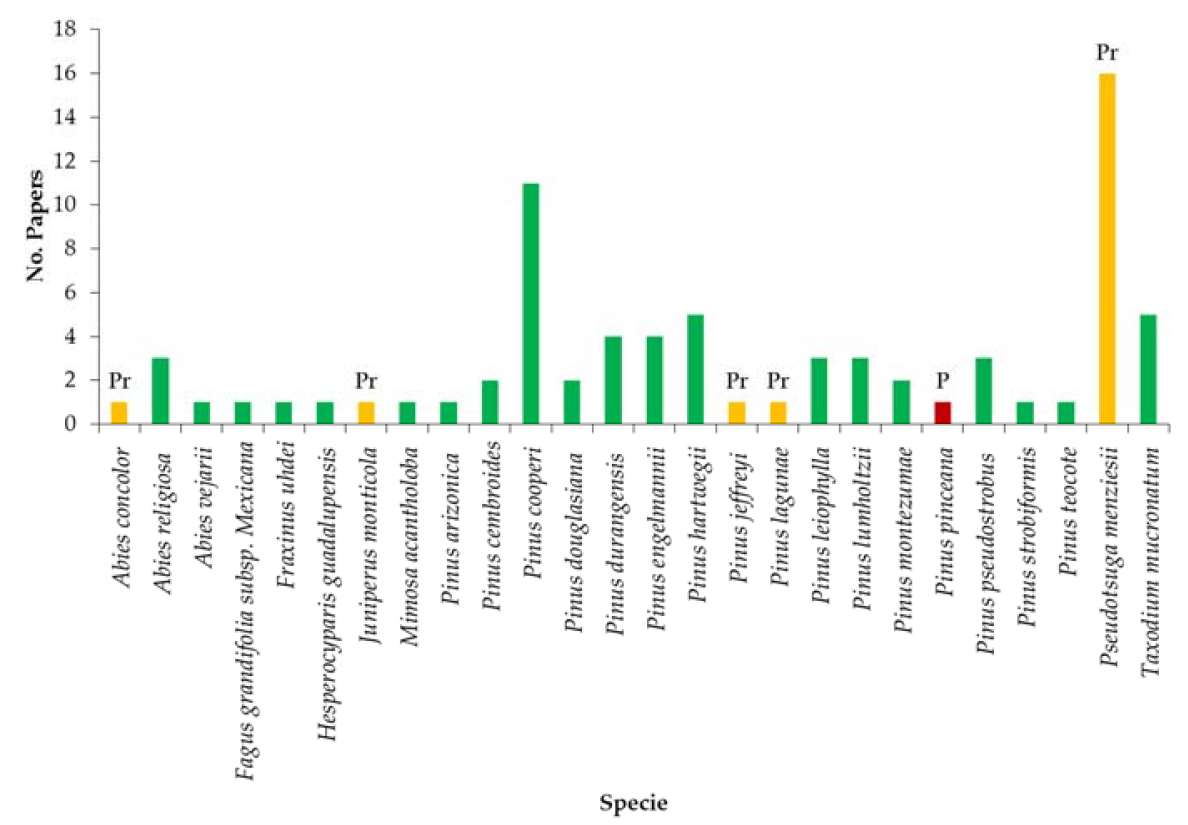

Figure 4. Number of dendrochronological studies carried out in Mexico grouped according to the sampled species and its conservation status. Yellow and red bars correspond to those trees species listed within a category in Mexican Official Standard 059 [28]. According to the Official Mexican Standard 059, " $\mathrm{Pr}^{\prime}$ indicates those tree species subject to special protection and " $\mathrm{P}$ " indicates endangered tree species.

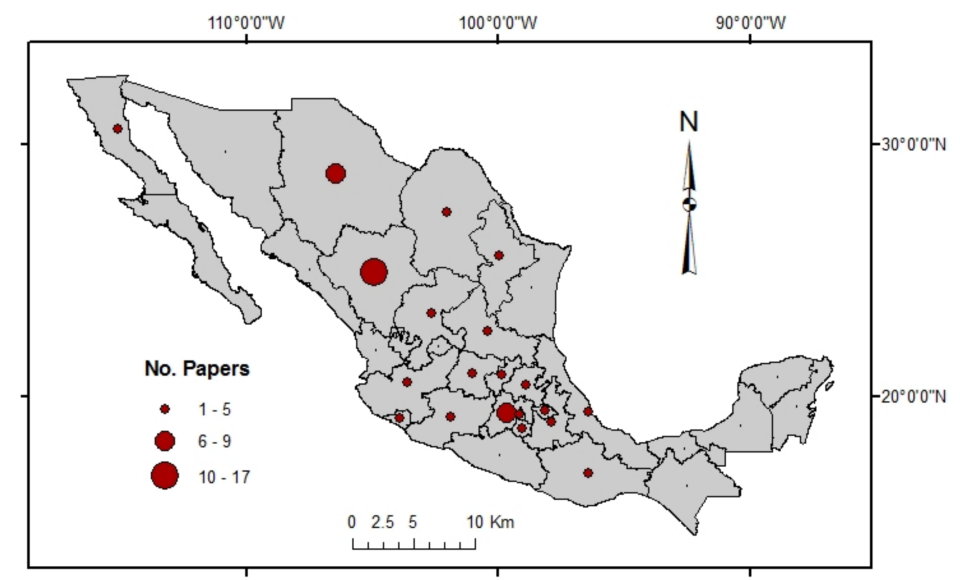

Figure 5. Dendrochronological studies performed in Mexico and grouped by states.

Regarding the temporary evolution, we observed an ascending tendency of the number of annual studies $(n=12)$ in the year 2016, with years 2003 and 2013 having more studies performed compared to the previous ones ( $n=4$ and 9 studies, respectively) (Figure 6).

In relation to the field of application, most of the studies have been developed with a climatic objective ( $n=30$ studies), usually for reconstructing precipitation. This is followed by those developed in the ecological ( $n=23$ studies) and hydrological ( $n=3$ studies) fields (Figure 7). A few studies were carried out in rarely explored fields, such as dendrogeomorphology or dendrochemistry (see Appendix A). 


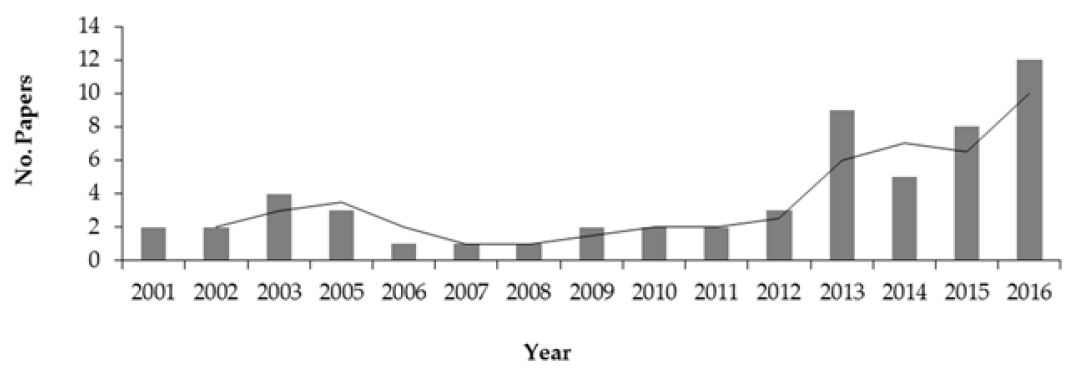

Figure 6. Dendrochronological studies realized in Mexico, grouped by year of publication.

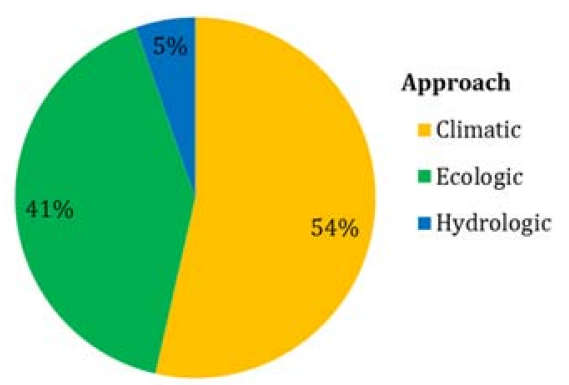

Figure 7. Dendrochronological studies performed in Mexico and grouped by its scope of application.

The journals in which the greatest numbers of Mexican dendrochronological studies have been published are mostly national journals covering $40 \%$ of the studies, such as: Madera y Bosques $(n=9$ studies), Revista Chapingo-Serie Ciencias Forestales y del Ambiente ( $n=5$ studies), Agrociencia $(n=4$ studies). This is followed by international journals, such as Dendrochronologia or Tree-Ring Research, which represent the remaining $60 \%$ of the studies (Figure 8).

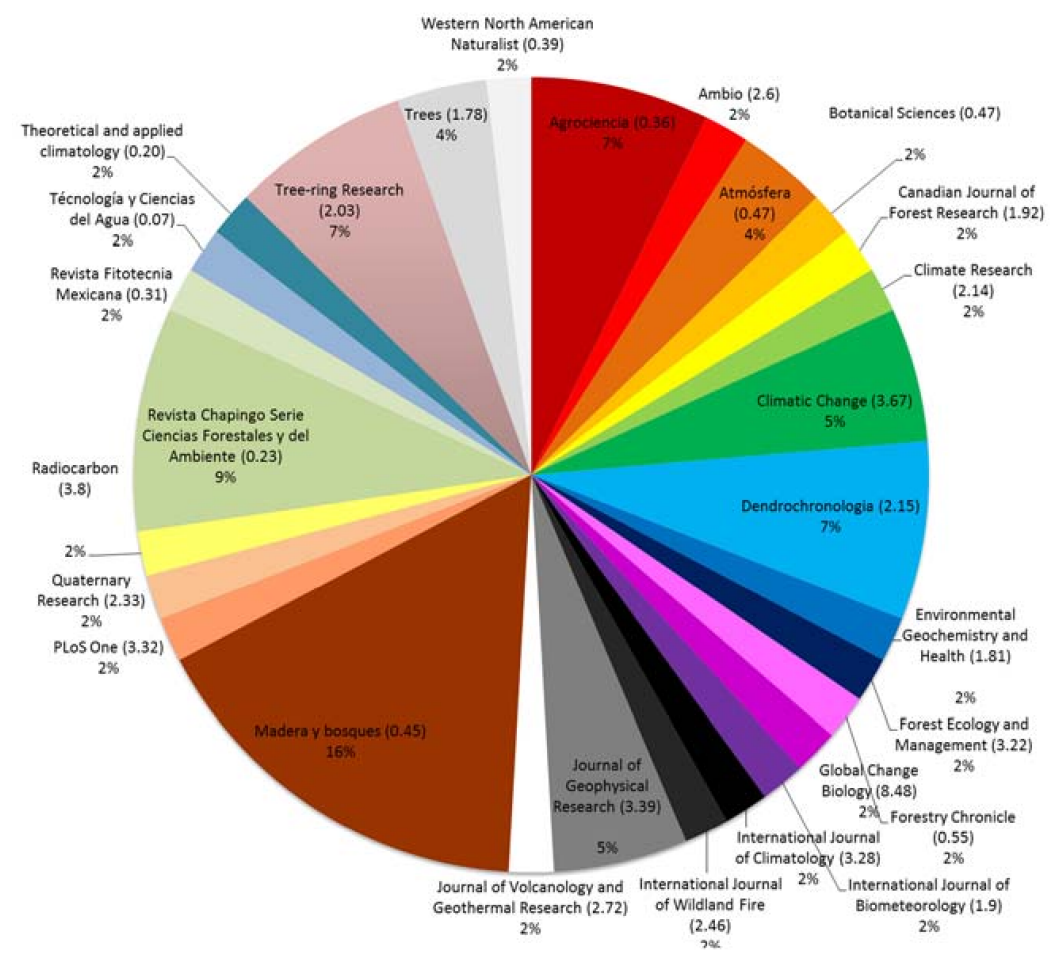

Figure 8. Dendrochronological studies on Mexican forests grouped according to the journal where they were published. The impact factor of each journal was estimated for 2015 citation data. 
Dendrochronological studies have been carried out including measures of width of earlywood (EW), latewood (LW) and tree-ring width (TRW). A total of 37 studies were found for the case of TRW, while 7 studies were detected for EW. For LW, only 1 study was found. Some studies analyzed several of these variables (Figure 9). A few studies considered other variables, such as carbon and oxygen isotopes, wood density or scars due to fires or volcanic activity (see Appendix A).

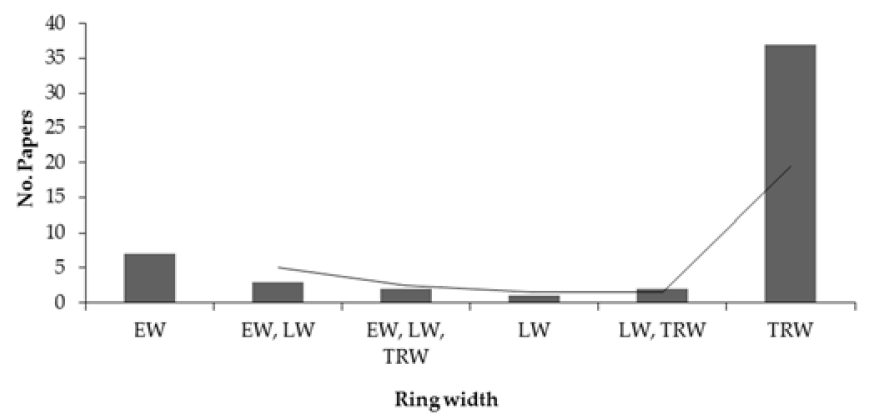

Figure 9. Dendrochronological studies performed in Mexico considering measurements of the width of earlywood (EW), latewood (LW) and total ring width (TRW).

The studies considered chronologies or mean series of tree-ring variables of different lengths or amplitudes, ranging from 49 to 607 years. Most studies are concentrated in an amplitude of 100 to 300 years (Figure 10). This is because some trees have sufficient longevity to contribute to the reconstruction of climatic events over a wide period of time. This is the case for the earlywood chronologies of up to 554 years of the extension of P. menziesii developed by Villanueva-Díaz et al. for temperate forests located in northern Mexico [24].

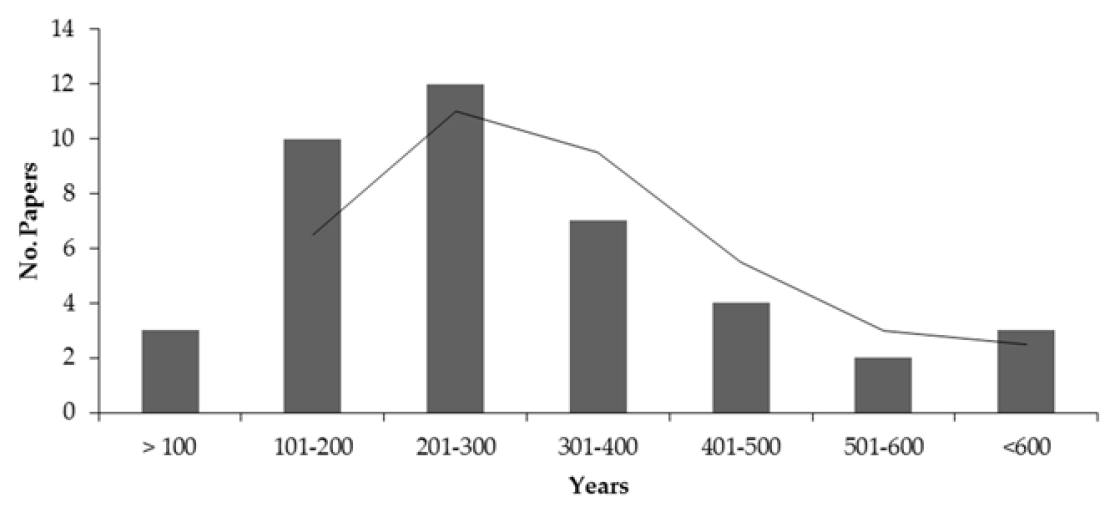

Figure 10. Dendrochronological studies performed in Mexico and grouped according to the amplitude or length of the chronologies.

The species that present a greater climatic sensitivity, quantified as correlations between climatic variables and tree-ring width, were P. menziesii, T. mucronatum and P. cooperi.

A total of $69 \%$ of the dendrochronological research was generated in Mexican institutions (Figure 11), with almost 60\% concentrated in two of the nine national centers where the research was carried out, namely the National Institute of Forest, Agriculture and Livestock Research (INIFAP) located in Gómez Palacio (Durango) and the Juarez University of Durango State [32,33]. Of the national institutions, $71 \%$ correspond to educational centers and 31\% to research centers. In Mexico, INIFAP is one of the few laboratories with a long tradition in dendrochronology. In other studies, such as Díaz et al. [34], Sheppard et al. [35] or González-Cásares et al. [36], some parts were conducted in overseas laboratories located mainly in United States of America (USA) and Europe. In USA, the universities of Northern Arizona, Arizona and Arkansas were the institutions in which a greater 
number of studies were carried out. The University of Arizona is considered as a pioneering center in conducting studies of this type in Mexico [8], although it has a smaller number of published studies compared to the University of Arkansas [37,38].

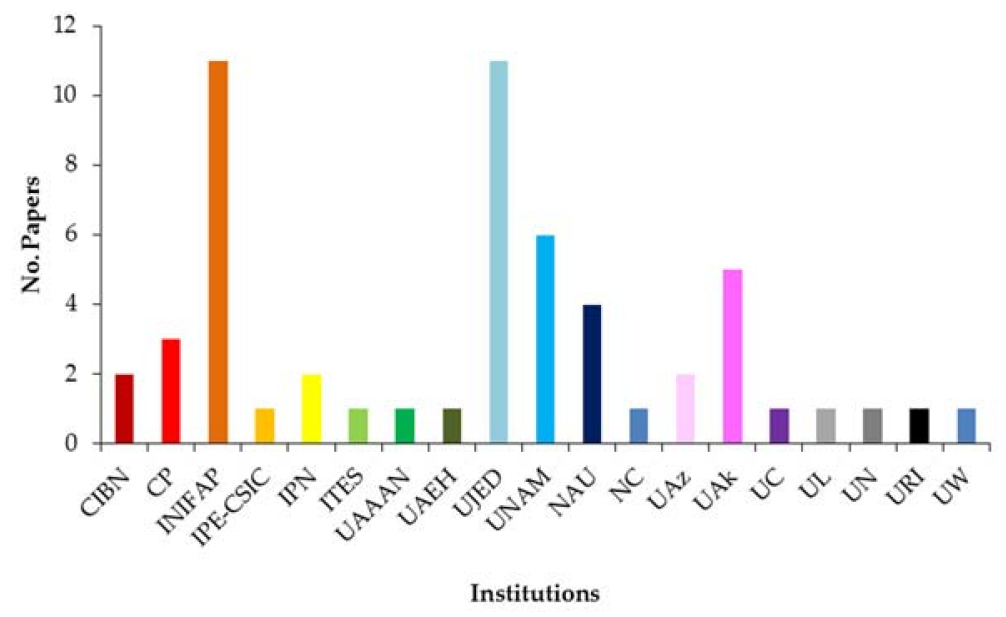

Figure 11. Research centers in which work is carried out around dendrochronological issues in Mexico estimated according to the first author of the study). The abbreviations are: National Center of Biological Research of the Northwest (CIBN), College of Postgraduates (CP), National Institute of Forestry, Agriculture and Livestock Research (INIFAP), Pyrenean Institute of Ecology (IPE-CSIC), National Politecnich Institute (IPN), Technological Institute of El Salto (ITES), Antonio Narro Autonomous Agrarian University (UAAAN), Autonomous University of Hidalgo State (UAEH), Juarez University of Durango State (UJED), National Autonomous University of Mexico (UNAM), Northern Arizona University (NAU), The Nature Conservancy (NC), University of Arizona (UAz), University of Arkansas (UAk), University of California (UC), University of Leeds (UL), University of Nevada (UN), University of Rhode Island (URI) and University of Washington (UW).

\section{Conclusions}

This is the first systematic review conducted for dendrochronological investigations carried out in Mexico that were published from 2001 until 2016 in indexed journals. Conifers are the most intensively sampled tree species because of their longevity and sensitivity to climate variability. Regarding the measured variables, most studies were based on the measurement of tree-ring width, although measuring earlywood and latewood features may also contribute to improving the reach of some studies. A lack of studies on tropical dry forests has been detected, which is an area of opportunity for the development of projects given the high richness of tree species found in this biome and because these forests experience seasonal droughts. There is a marked tendency in carrying out tree-ring studies focusing on climatic reconstructions, which suggests that it may be beneficial to broaden tree-ring sciences to encompass other research fields, such as ecology (dendroecology) or geomorphology (dendrogeomorphology). Dendrochronological studies are mostly published in international journals. Collaboration with national and international researchers is a great opportunity to promote the future development of dendrochronology in Mexico.

Acknowledgments: Funding was provided by CONACYT (Consejo Nacional de Ciencia y Tecnología) through the CB-2013/222522 project. Authors are grateful to Editors and anonymous reviewers for their useful comments and suggestions.

Author Contributions: M.P.-G. and J.J.C. conceived and designed the review; A.C.A.-H. performed the review; all authors analyzed the data and wrote the paper.

Conflicts of Interest: The authors declare no conflict of interest. 


\section{Appendix A}

Table A1. List of articles consulted on dendrochronology by species or Mexican forests published in indexed scientific journals.

\begin{tabular}{|c|c|c|c|c|c|c|c|c|c|c|}
\hline No & Year & Journal & Authors & Title & Family & Tree Species & $\begin{array}{c}\text { Ecosystem } \\
\text { (INEGI } \\
\text { Classification) } \\
\end{array}$ & Region/State & $\begin{array}{c}\text { Area of } \\
\text { Application }\end{array}$ & Variable \\
\hline 1 & 2001 & Ambio & Biondi, $\mathrm{F}$. & $\begin{array}{l}\text { A 400-year tree-ring } \\
\text { chronology from the } \\
\text { tropical treeline of } \\
\text { North America. }\end{array}$ & Pinaceae & Pinus hartwegii Lindl. & Pine forest & Colima & Dendroclimatology & TRW \\
\hline 2 & 2001 & $\begin{array}{l}\text { International } \\
\text { Journal of } \\
\text { Climatology }\end{array}$ & $\begin{array}{l}\text { Díaz, S.C.; Touchan, R.; } \\
\text { Swetnam, T. }\end{array}$ & $\begin{array}{l}\text { A tree-ring } \\
\text { reconstruction of past } \\
\text { precipitation for Baja } \\
\text { California Sur, Mexico. }\end{array}$ & Pinaceae & $\begin{array}{l}\text { Pseudotsuga menziesii } \\
\text { (Mirb.) Franco }\end{array}$ & Pine-oak forest & $\begin{array}{c}\text { Baja } \\
\text { California }\end{array}$ & Dendroclimatology & TRW \\
\hline 3 & 2002 & $\begin{array}{l}\text { Climate } \\
\text { Research }\end{array}$ & $\begin{array}{l}\text { Díaz, S.C.; Therrell, M.; } \\
\text { Stahle, D.; Cleaveland, M. }\end{array}$ & $\begin{array}{l}\text { Chihuahua (Mexico) } \\
\text { winter-spring } \\
\text { precipitation } \\
\text { reconstructed from } \\
\text { tree-rings, 1647-1992. }\end{array}$ & Pinaceae & $\begin{array}{l}\text { Pinus lagunae } \\
\text { (Rob.-Pass.) Passini }\end{array}$ & Oyamel forest & $\begin{array}{l}\text { Chihuahua } \\
\text { and Durango }\end{array}$ & Dendroclimatology & EW \\
\hline 4 & 2002 & $\begin{array}{l}\text { Journal of } \\
\text { Geophysical } \\
\text { Research }\end{array}$ & $\begin{array}{l}\text { Therrell, M.; Stahle, D.; } \\
\text { Cleaveland, M.; } \\
\text { Villanueva-Díaz, J. }\end{array}$ & $\begin{array}{l}\text { Warm season tree } \\
\text { growth and } \\
\text { precipitation over } \\
\text { Mexico. }\end{array}$ & $\begin{array}{l}\text { Pinaceae, } \\
\text { Cupressaceae }\end{array}$ & $\begin{array}{l}\text { Pseudotsuga menziesii } \\
\text { (Mirb.) Franco, } \\
\text { Pinus montezumae } \\
\text { Lamb., } \\
\text { Taxodium mucronatum } \\
\text { Ten. }\end{array}$ & $\begin{array}{l}\text { Pine forest and } \\
\text { Gallery forest }\end{array}$ & - & Dendroclimatology & $\begin{array}{l}\text { LW and } \\
\text { TRW }\end{array}$ \\
\hline 5 & 2003 & $\begin{array}{l}\text { Tree-ring } \\
\text { Research }\end{array}$ & $\begin{array}{l}\text { Pohl, K.; Therrell, M.; Blay, } \\
\text { J.; Ayotte, A.; Cabrera, J.; } \\
\text { Díaz, S.; Cornejo, E.; Elvir, J.; } \\
\text { González, M.; Opland, D.; } \\
\text { Park, J.; Pederson, G.; } \\
\text { Bernal, S.; Vázquez, L.; } \\
\text { Villanueva-Díaz, J.; } \\
\text { Stahle, D. }\end{array}$ & $\begin{array}{l}\text { A cool season } \\
\text { precipitation } \\
\text { reconstruction for } \\
\text { Saltillo, Mexico. }\end{array}$ & Pinaceae & $\begin{array}{l}\text { Pseudotsuga menziesii } \\
\text { (Mirb.) Franco }\end{array}$ & Oyamel forest & Coahuila & Dendroclimatology & EW \\
\hline 6 & 2003 & $\begin{array}{l}\text { Climatic } \\
\text { Change }\end{array}$ & $\begin{array}{l}\text { Cleaveland, M.; Stahle, D.; } \\
\text { Therrell, M.; } \\
\text { Villanueva-Diaz, J.; } \\
\text { Burns, B. }\end{array}$ & $\begin{array}{l}\text { Tree-Ring } \\
\text { Reconstructed Winter } \\
\text { Precipitation and } \\
\text { Tropical } \\
\text { Teleconnections in } \\
\text { Durango, Mexico. }\end{array}$ & Pinaceae & $\begin{array}{l}\text { Pseudotsuga menziesii } \\
\text { (Mirb.) Franco }\end{array}$ & Oyamel forest & Durango & Dendroclimatology & EW \\
\hline 7 & 2003 & $\begin{array}{l}\text { Canadian } \\
\text { Journal of } \\
\text { Forest Research }\end{array}$ & $\begin{array}{l}\text { Stephens, S.; Skinner, C.; } \\
\text { Gill, S. }\end{array}$ & $\begin{array}{l}\text { Dendrochronology-based } \\
\text { fire history of Jeffrey } \\
\text { pine-mixed conifer } \\
\text { forests in the Sierra San } \\
\text { Pedro Martir, Mexico. }\end{array}$ & Pinaceae & Pinus jeffreyi Balf. & Pine forest & $\begin{array}{c}\text { Baja } \\
\text { California }\end{array}$ & Dendroecology & Fire scars \\
\hline
\end{tabular}


Table A1. Cont.

\begin{tabular}{|c|c|c|c|c|c|c|c|c|c|c|}
\hline No & Year & Journal & Authors & Title & Family & Tree Species & $\begin{array}{c}\text { Ecosystem } \\
\text { (INEGI } \\
\text { Classification) }\end{array}$ & Region/State & $\begin{array}{c}\text { Area of } \\
\text { Application }\end{array}$ & Variable \\
\hline 8 & 2003 & $\begin{array}{l}\text { Quaternary } \\
\text { Research }\end{array}$ & $\begin{array}{l}\text { Biondi, F; Galindo I.; } \\
\text { Gavilanes, J.; Elizalde, A. }\end{array}$ & $\begin{array}{l}\text { Tree growth response } \\
\text { to the } 1913 \text { eruption of } \\
\text { Volcán de Fuego de } \\
\text { Colima, Mexico. }\end{array}$ & Pinaceae & Pinus hartwegii Lindl. & Pine forest & Colima & Dendroecology & TRW \\
\hline 9 & 2005 & $\begin{array}{l}\text { Forest Ecology } \\
\text { and } \\
\text { Management }\end{array}$ & $\begin{array}{l}\text { González-Elizondo, M.; } \\
\text { Jurado, E.; Návar, J.; } \\
\text { González-Elizondo, M.S.; } \\
\text { Villanueva, J.; Aguirre, O.; } \\
\text { Jiménez, J. }\end{array}$ & $\begin{array}{l}\text { Tree-rings and climate } \\
\text { relationships for } \\
\text { Douglas-fir } \\
\text { chronologies from the } \\
\text { Sierra Madre } \\
\text { Occidental, Mexico: A } \\
\text { 1681-2001 rain } \\
\text { reconstruction. }\end{array}$ & Pinaceae & $\begin{array}{l}\text { Pseudotsuga menziesii } \\
\text { (Mirb.) Franco }\end{array}$ & Oyamel forest & $\begin{array}{l}\text { Durango and } \\
\text { Zacatecas }\end{array}$ & Dendroclimatology & TRW \\
\hline 10 & 2005 & Dendrochronologia & Therrell, M. & $\begin{array}{l}\text { Tree rings and "El año } \\
\text { del hambre" in Mexico. }\end{array}$ & Pinaceae & & Oyamel forest & $\begin{array}{c}\text { Durango and } \\
\text { Zacatecas }\end{array}$ & Dendroclimatology & \\
\hline 11 & 2005 & Dendrochronologia & $\begin{array}{l}\text { Villanueva, J.; Luckman, B.; } \\
\text { Stahle, D.; Therrell, M.; } \\
\text { Cleaveland, M.; } \\
\text { Cerano-Paredes, J.; } \\
\text { Gutierrez-Garcia, G.; } \\
\text { Estrada-Avalos, J.; } \\
\text { Jasso-Ibarra, R. }\end{array}$ & $\begin{array}{l}\text { Hydroclimatic } \\
\text { variability of the upper } \\
\text { Nazas basin: water } \\
\text { management } \\
\text { implications for the } \\
\text { irrigated area of the } \\
\text { Comarca Lagunera. }\end{array}$ & Pinaceae & $\begin{array}{l}\text { Pseudotsuga menziesii } \\
\text { (Mirb.) Franco, } \\
\text { Pinus durangensis } \\
\text { Martínez }\end{array}$ & Pine forest & $\begin{array}{l}\text { Durango and } \\
\text { Zacatecas }\end{array}$ & Dendrohydrology & EW \\
\hline 12 & 2006 & $\begin{array}{l}\text { Climatic } \\
\text { Change }\end{array}$ & $\begin{array}{l}\text { Therrell, M.; Stahle, D.; } \\
\text { Villanueva-Díaz, J.; } \\
\text { Cornejo-Oviedo, E.; } \\
\text { Cleaveland, M. }\end{array}$ & $\begin{array}{l}\text { Tree-ring reconstructed } \\
\text { maize yield in central } \\
\text { Mexico: } 1474-2001 .\end{array}$ & Pinaceae & $\begin{array}{l}\text { Pseudotsuga menziesii } \\
\text { (Mirb.) Franco }\end{array}$ & Oyamel forest & Puebla & Dendroecology & LW \\
\hline 13 & 2007 & $\begin{array}{l}\text { Climatic } \\
\text { Change }\end{array}$ & $\begin{array}{l}\text { Villanueva-Diaz, J.; } \\
\text { Stahle, D.; Luckman, B.; } \\
\text { Cerano-Paredes, J.; } \\
\text { Therrell, M.; } \\
\text { Cleaveland, M.; } \\
\text { Cornejo-Oviedo, E. }\end{array}$ & $\begin{array}{l}\text { Winter-spring } \\
\text { precipitation } \\
\text { reconstructions from } \\
\text { tree rings for northeast } \\
\text { México. }\end{array}$ & Pinaceae & $\begin{array}{l}\text { Pseudotsuga menziesii } \\
\text { (Mirb.) Franco }\end{array}$ & Oyamel forest & $\begin{array}{l}\text { Coahuila, } \\
\text { Nuevo León } \\
\text { and } \\
\text { Tamaulipas }\end{array}$ & Dendroclimatology & EW \\
\hline 14 & 2008 & $\begin{array}{l}\text { Tree-ring } \\
\text { Research }\end{array}$ & $\begin{array}{l}\text { Sheppard, P.; Ort, M.; } \\
\text { Anderson, K.; Elson, M.; } \\
\text { Vazquez-Salem, L.; } \\
\text { Clemens, A.; Little, N.; } \\
\text { Speakman, R. }\end{array}$ & $\begin{array}{l}\text { Multiple } \\
\text { dendrochronological } \\
\text { signals indicate the } \\
\text { eruption of Paricutín } \\
\text { volcano, Michoacán, } \\
\text { México. }\end{array}$ & Pinaceae & $\begin{array}{l}\text { Pinus leiophylla Schiede } \\
\text { ex Schltdl. \& Cham., } \\
\text { Pinus pseudostrobus } \\
\text { Lindl., } \\
\text { Pinus montezumae } \\
\text { Lamb., Pinus teocote } \\
\text { Schltdl. \& Cham. }\end{array}$ & Pine forest & Michoacan & Dendroecology & $\begin{array}{l}\text { LW and } \\
\text { TRW }\end{array}$ \\
\hline 15 & 2009 & $\begin{array}{l}\text { Madera y } \\
\text { bosques }\end{array}$ & $\begin{array}{l}\text { Villanueva Díaz, J.; } \\
\text { Cerano Paredes, J.; } \\
\text { Constante-García, V.; } \\
\text { Fulé, P.; Cornejo, E. }\end{array}$ & $\begin{array}{l}\text { Variabilidad } \\
\text { hidroclimática } \\
\text { histórica de la sierra de } \\
\text { Zapalinamé y } \\
\text { disponibilidad de } \\
\text { recursos hídricos para } \\
\text { Saltillo, Coahuila. }\end{array}$ & Pinaceae & $\begin{array}{l}\text { Pseudotsuga menziesii } \\
\text { (Mirb.) Franco, } \\
\text { Pinus cembroides } \\
\text { Gordon }\end{array}$ & Pine forest & $\begin{array}{l}\text { Coahuila and } \\
\text { Nuevo León }\end{array}$ & $\begin{array}{l}\text { Dendroclimatology } \\
\text { and } \\
\text { dendrohydrology }\end{array}$ & $\begin{array}{l}\text { EW, LW } \\
\text { and TRW }\end{array}$ \\
\hline
\end{tabular}


Table A1. Cont.

\begin{tabular}{|c|c|c|c|c|c|c|c|c|c|c|}
\hline No & Year & Journal & Authors & Title & Family & Tree Species & $\begin{array}{c}\text { Ecosystem } \\
\text { (INEGI } \\
\text { Classification) }\end{array}$ & Region/State & $\begin{array}{c}\text { Area of } \\
\text { Application }\end{array}$ & Variable \\
\hline 16 & 2009 & $\begin{array}{l}\text { Madera y } \\
\text { bosques }\end{array}$ & $\begin{array}{l}\text { Cerano, J.; Villanueva, J.; } \\
\text { Fulé, P.; Arreola, J.; } \\
\text { Sánchez, I.; Valdez, R. }\end{array}$ & $\begin{array}{l}\text { Reconstrucción de } 350 \\
\text { años de precipitación } \\
\text { para el suroeste de } \\
\text { Chihuahua, México. }\end{array}$ & Pinaceae & $\begin{array}{l}\text { Pseudotsuga menziesii } \\
\text { (Mirb.) Franco }\end{array}$ & Oyamel forest & Chihuahua & Dendroclimatology & $\begin{array}{l}\text { EW, LW } \\
\text { and TRW }\end{array}$ \\
\hline 17 & 2010 & $\begin{array}{l}\text { Madera y } \\
\text { bosques }\end{array}$ & $\begin{array}{l}\text { Arreola-Ortiz, M.; } \\
\text { González-Elizondo, M.; } \\
\text { Návar-Cháidez, J. }\end{array}$ & $\begin{array}{l}\text { Dendrocronología de } \\
\text { Pseudotsuga menziesii } \\
\text { (Mirb.) Franco de la } \\
\text { Sierra Madre Oriental } \\
\text { en Nuevo León, } \\
\text { México. }\end{array}$ & Pinaceae & $\begin{array}{l}\text { Pseudotsuga menziesii } \\
\text { (Mirb.) Franco }\end{array}$ & Oyamel forest & Nuevo León & Dendroclimatology & TRW \\
\hline 18 & 2010 & $\begin{array}{l}\text { Madera y } \\
\text { bosques }\end{array}$ & $\begin{array}{l}\text { Santillán-Hernández, M.; } \\
\text { Cornejo-Oviedo, E.; } \\
\text { Villanueva-Díaz, J.; } \\
\text { Cerano-Paredes, J.; } \\
\text { Valencia-Manzo, S.; } \\
\text { Capó-Arteaga, M. } \\
\end{array}$ & $\begin{array}{l}\text { Potencial } \\
\text { dendroclimático de } \\
\text { Pinus pinceana Gordon } \\
\text { en la Sierra Madre } \\
\text { Oriental }\end{array}$ & Pinaceae & $\begin{array}{l}\text { Pinus pinceana Gordon } \\
\text { \& Glend. }\end{array}$ & Pine forest & $\begin{array}{l}\text { Hidalgo, } \\
\text { Queretaro, } \\
\text { Zacatecas, } \\
\text { San Luis } \\
\text { Potosi and } \\
\text { Coahuila } \\
\end{array}$ & Dendroclimatology & TRW \\
\hline 19 & 2011 & $\begin{array}{l}\text { Western North } \\
\text { American } \\
\text { Naturalist }\end{array}$ & Bickford, I.; Fulé, P.; Kolb, T. & $\begin{array}{l}\text { Growth Sensitivity to } \\
\text { Drought of } \\
\text { Co-Occurring Pinus } \\
\text { spp. along an } \\
\text { Elevation Gradient in } \\
\text { Northern Mexico. }\end{array}$ & Pinaceae & $\begin{array}{l}\text { Pinus engelmannii } \\
\text { Carrière, } \\
\text { Pinus lumholtzii B.L. } \\
\text { Rob. \& Fernald }\end{array}$ & Pine forest & Chihuahua & Dendroecology & TRW \\
\hline 20 & 2011 & $\begin{array}{l}\text { Revista } \\
\text { Chapingo Serie } \\
\text { Ciencias } \\
\text { Forestales y del } \\
\text { Ambiente }\end{array}$ & $\begin{array}{l}\text { Cerano-Paredes, J.; } \\
\text { Villanueva-Díaz, J.; } \\
\text { Valdez-Cepeda, R.; } \\
\text { Arreola-Ávila, J.; } \\
\text { Constante-García, V. }\end{array}$ & $\begin{array}{l}\text { El Niño Oscilación del } \\
\text { Sur y sus efectos en la } \\
\text { precipitación en la } \\
\text { parte alta de la cuenca } \\
\text { del río Nazas. }\end{array}$ & Pinaceae & $\begin{array}{l}\text { Pseudotsuga menziesii } \\
\text { (Mirb.) Franco }\end{array}$ & Oyamel forest & Durango & Dendrohydrology & TRW \\
\hline 21 & 2012 & $\begin{array}{l}\text { Técnología y } \\
\text { Ciencias del } \\
\text { Agua }\end{array}$ & $\begin{array}{l}\text { Villanueva-Díaz, J.; } \\
\text { Cerano-Paredes, J.; } \\
\text { Estrada-Ávalos, J.; } \\
\text { Constante-García, V.; } \\
\text { Cortés-Barrera, E. }\end{array}$ & $\begin{array}{l}\text { Variabilidad } \\
\text { hidroclimática } \\
\text { reconstruida con } \\
\text { anillos de árboles para } \\
\text { la cuenca Lerma } \\
\text { Chapala en } \\
\text { Guanajuato, México. }\end{array}$ & $\begin{array}{l}\text { Pinaceae, } \\
\text { Cupressaceae }\end{array}$ & $\begin{array}{l}\text { Taxodium mucronatum } \\
\text { Ten., Pinus cembroides } \\
\text { Gordon }\end{array}$ & $\begin{array}{l}\text { Pine forest and } \\
\text { Gallery forest }\end{array}$ & $\begin{array}{l}\text { Guanajuato, } \\
\text { Jalisco and } \\
\text { Queretaro }\end{array}$ & Dendroclimatology & TRW \\
\hline 22 & 2012 & $\begin{array}{l}\text { Revista } \\
\text { Chapingo Serie } \\
\text { Ciencias } \\
\text { Forestales y del } \\
\text { Ambiente }\end{array}$ & $\begin{array}{l}\text { Villanueva Díaz, J.; Fulé, P.; } \\
\text { Cerano Paredes, J.; Estrada } \\
\text { Avalos, J.; Sánchez Cohen, I. }\end{array}$ & $\begin{array}{l}\text { Reconstrucción de } \\
\text { precipitación } \\
\text { estacional para el } \\
\text { barlovento de la Sierra } \\
\text { Madre Occidental. }\end{array}$ & Pinaceae & $\begin{array}{l}\text { Pseudotsuga menziesii } \\
\text { (Mirb.) Franco }\end{array}$ & Oyamel forest & Durango & Dendroclimatology & EW \\
\hline 23 & 2012 & $\begin{array}{l}\text { Forestry } \\
\text { Chronicle }\end{array}$ & Cassell, B.; Alvarado, E. & $\begin{array}{l}\text { Reconstruction of fire } \\
\text { history in Mexican } \\
\text { tropical pines using } \\
\text { tree rings. }\end{array}$ & Pinaceae & $\begin{array}{l}\text { Pinus douglasiana } \\
\text { Martínez }\end{array}$ & Pine forest & Jalisco & Dendroecology & $\begin{array}{l}\text { TRW, fire } \\
\text { scars }\end{array}$ \\
\hline
\end{tabular}


Table A1. Cont.

\begin{tabular}{|c|c|c|c|c|c|c|c|c|c|c|}
\hline No & Year & Journal & Authors & Title & Family & Tree Species & $\begin{array}{c}\text { Ecosystem } \\
\text { (INEGI } \\
\text { Classification) }\end{array}$ & Region/State & $\begin{array}{c}\text { Area of } \\
\text { Application }\end{array}$ & Variable \\
\hline 24 & 2013 & $\begin{array}{l}\text { Revista } \\
\text { Chapingo Serie } \\
\text { Ciencias } \\
\text { Forestales y del } \\
\text { Ambiente }\end{array}$ & $\begin{array}{l}\text { Cerano-Paredes, J.; } \\
\text { Méndez-González, J.; } \\
\text { Amaro-Sánchez, A.; } \\
\text { Villanueva-Díaz, J.; } \\
\text { Cervantes-Martínez, R.; } \\
\text { Rubio-Camacho, E. }\end{array}$ & $\begin{array}{l}\text { Reconstrucción de } \\
\text { precipitación } \\
\text { invierno-primavera } \\
\text { con anillos anuales de } \\
\text { Pinus douglasiana en la } \\
\text { Reserva de la Biosfera } \\
\text { Sierra de Manantlán, } \\
\text { Jalisco. }\end{array}$ & Pinaceae & $\begin{array}{l}\text { Pinus douglasiana } \\
\text { Martínez }\end{array}$ & Pine forest & Jalisco & Dendroclimatology & TRW \\
\hline 25 & 2013 & Dendrochronologia & $\begin{array}{l}\text { Pompa-García, M.; } \\
\text { Cerano-Paredes, J.; Fulé, P. }\end{array}$ & $\begin{array}{l}\text { Variation in radial } \\
\text { growth of Pinus cooperi } \\
\text { in response to climatic } \\
\text { signals across an } \\
\text { elevational gradient. }\end{array}$ & Pinaceae & $\begin{array}{l}\text { Pinus cooperi C.E. } \\
\text { Blanco }\end{array}$ & Pine forest & Durango & Dendroecology & TRW \\
\hline 26 & 2013 & Agrociencia & $\begin{array}{l}\text { Pompa-García, M.; } \\
\text { Rodríguez-Flores, F; } \\
\text { Aguirre-Salado, C.; } \\
\text { Miranda-Aragón, L. }\end{array}$ & $\begin{array}{l}\text { Influencia de la } \\
\text { evaporación en el } \\
\text { crecimiento forestal. }\end{array}$ & Pinaceae & $\begin{array}{l}\text { Pinus cooperi C.E. } \\
\text { Blanco }\end{array}$ & Pine forest & Durango & Dendroecology & TRW \\
\hline 27 & 2013 & $\begin{array}{l}\text { Madera y } \\
\text { bosques }\end{array}$ & $\begin{array}{l}\text { Irby, C.; Fulé, P.; Yocom, L.; } \\
\text { Villanueva, J. }\end{array}$ & $\begin{array}{l}\text { Dendrochronological } \\
\text { reconstruction of } \\
\text { long-term precipitation } \\
\text { patterns in Basaseachi } \\
\text { National Park, } \\
\text { Chihuahua, Mexico. }\end{array}$ & Pinaceae & $\begin{array}{l}\text { Pinus durangensis } \\
\text { Martínez, } \\
\text { Pinus lumholtzii B.L. } \\
\text { Rob. \& Fernald, } \\
\text { Pinus engelmannii } \\
\text { Carrière }\end{array}$ & Pine forest & Chihuahua & Dendroclimatology & TRW \\
\hline 28 & 2013 & $\begin{array}{l}\text { Journal of } \\
\text { Volcanology } \\
\text { and Geothermal } \\
\text { Research }\end{array}$ & $\begin{array}{l}\text { Franco-Ramos, O.; } \\
\text { Stoffel, M.; } \\
\text { Vázquez-Selem, L.; } \\
\text { Capra, L. }\end{array}$ & $\begin{array}{l}\text { Spatio-temporal } \\
\text { reconstruction of } \\
\text { lahars on the southern } \\
\text { slopes of Colima } \\
\text { volcano, Mexico-A } \\
\text { dendrogeomorphic } \\
\text { approach. }\end{array}$ & Pinaceae & $\begin{array}{l}\text { Pinus leiophylla Schiede } \\
\text { ex Schltdl. \& Cham. }\end{array}$ & Pine forest & Colima & Dendroecology & TRW, sacrs \\
\hline 29 & 2013 & Radiocarbon & $\begin{array}{l}\text { Beramendi-Orosco, L.; } \\
\text { Hernandez-Morales, S.; } \\
\text { Gonzalez-Hernandez, G.; } \\
\text { Constante-Garcia, V.; } \\
\text { Villanueva-Diaz, J. }\end{array}$ & $\begin{array}{l}\text { Dendrochronological } \\
\text { potential of Fraxinus } \\
\text { uhdei and its use as } \\
\text { bioindicator of fossil } \\
\text { CO2 emissions } \\
\text { deduced from } \\
\text { radiocarbon } \\
\text { concentrations in tree } \\
\text { rings. }\end{array}$ & Oleaceae & $\begin{array}{l}\text { Fraxinus uhdei (Wenz.) } \\
\text { Lingelsh. }\end{array}$ & $\begin{array}{l}\text { Subtropical } \\
\text { scrubland }\end{array}$ & $\begin{array}{l}\text { San Luis } \\
\text { Potosi }\end{array}$ & Dendroecology & TRW \\
\hline 30 & 2013 & $\begin{array}{l}\text { Global Change } \\
\text { Biology }\end{array}$ & $\begin{array}{l}\text { Gómez-Guerrero, A.; } \\
\text { Silva, L.; Barrera-Reyes, M.; } \\
\text { Kishchuk, B.; } \\
\text { Velázquez-Martínez, A.; } \\
\text { Martínez-Trinidad, T.; } \\
\text { Plascencia-Escalante, F.; } \\
\text { Horwath, W. }\end{array}$ & $\begin{array}{l}\text { Growth decline and } \\
\text { divergent tree ring } \\
\text { isotopic composition } \\
\left(\delta^{13} \mathrm{C} \text { and } \delta^{18} \mathrm{O}\right) \\
\text { contradict predictions } \\
\text { of } \mathrm{CO}_{2} \text { stimulation in } \\
\text { high altitudinal forests. }\end{array}$ & Pinaceae & $\begin{array}{l}\text { Abies religiosa (Kunth) } \\
\text { Schltdl. \& Cham., } \\
\text { Pinus hartwegii Lindl. }\end{array}$ & $\begin{array}{l}\text { Pine forest, } \\
\text { Oyamel forest }\end{array}$ & $\begin{array}{l}\text { Colima, } \\
\text { Michoacan, } \\
\text { Estado de } \\
\text { México, } \\
\text { Tlaxcala and } \\
\text { Veracruz }\end{array}$ & Dendroecology & $\begin{array}{c}\text { TRW, } \\
\text { carbon and } \\
\text { oxygen } \\
\text { isotopes }\end{array}$ \\
\hline
\end{tabular}


Table A1. Cont.

\begin{tabular}{|c|c|c|c|c|c|c|c|c|c|c|}
\hline No & Year & Journal & Authors & Title & Family & Tree Species & $\begin{array}{c}\text { Ecosystem } \\
\text { (INEGI } \\
\text { Classification) }\end{array}$ & Region/State & $\begin{array}{c}\text { Area of } \\
\text { Application }\end{array}$ & Variable \\
\hline 31 & 2013 & $\begin{array}{l}\text { Journal of } \\
\text { Geophysical } \\
\text { Research }\end{array}$ & $\begin{array}{l}\text { Brienen, R.; Hietz, P.; } \\
\text { Wanek, W.; Gloor, M. }\end{array}$ & $\begin{array}{l}\text { Oxygen isotopes in tree } \\
\text { rings record variation } \\
\text { in precipitation } \delta^{18} \mathrm{O} \\
\text { and amount effects in } \\
\text { the south of Mexico. }\end{array}$ & Fabaceae & $\begin{array}{l}\text { Mimosa acantholoba } \\
\text { (Humb. \& Bonpl. ex } \\
\text { Willd.) Poir. }\end{array}$ & & Oaxaca & Dendroclimatology & $\begin{array}{c}\text { TRW, } \\
\text { oxygen } \\
\text { isotopoes }\end{array}$ \\
\hline 32 & 2013 & $\begin{array}{l}\text { Journal of } \\
\text { Geophysical } \\
\text { Research }\end{array}$ & $\begin{array}{l}\text { Meko, D.; Touchan, R.; } \\
\text { Villanueva, J.; Griffin, D.; } \\
\text { Woodhouse, C.; Castro, C.; } \\
\text { Carillo, C.; Leavitt, S. }\end{array}$ & $\begin{array}{l}\text { Sierra San Pedro } \\
\text { Mártir, Baja California, } \\
\text { cool-season } \\
\text { precipitation } \\
\text { reconstructed from } \\
\text { earlywood width of } \\
\text { Abies concolor tree } \\
\text { rings. }\end{array}$ & Pinaceae & Abies concolor Lindl. & Oyamel forest & $\begin{array}{c}\text { Baja } \\
\text { California }\end{array}$ & Dendroclimatology & EW \\
\hline 33 & 2014 & $\begin{array}{l}\text { Theoretical and } \\
\text { Applied } \\
\text { Climatology }\end{array}$ & $\begin{array}{l}\text { Pompa-García, M.; } \\
\text { Jurado, E. }\end{array}$ & $\begin{array}{l}\text { Seasonal precipitation } \\
\text { reconstruction and } \\
\text { teleconnections with } \\
\text { ENSO based on tree } \\
\text { ring analysis of } \\
\text { Pinus cooperi. }\end{array}$ & Pinaceae & $\begin{array}{l}\text { Pinus cooperi C.E. } \\
\text { Blanco }\end{array}$ & Pine forest & Durango & Dendroclimatology & TRW \\
\hline 34 & 2014 & Agrociencia & $\begin{array}{l}\text { Villanueva-Díaz, J.; } \\
\text { Cerano-Paredes, J.; } \\
\text { Gómez-Guerrero, A.; } \\
\text { Correa-Díaz, A.; } \\
\text { Castruita-Esparza, L.; } \\
\text { Cervantes-Martínez, R.; } \\
\text { Stahle, D.; } \\
\text { Martínez-Sifuentes, A. }\end{array}$ & $\begin{array}{l}\text { Cinco siglos de historia } \\
\text { dendrocronológica de } \\
\text { los ahuehuetes } \\
\text { (Taxodium mucronatum } \\
\text { Ten.) del Parque el } \\
\text { Contador, San } \\
\text { Salvador Atenco, } \\
\text { Estado de México. }\end{array}$ & Cupressaceae & $\begin{array}{l}\text { Taxodium mucronatum } \\
\text { Ten. }\end{array}$ & Gallery forest & $\begin{array}{l}\text { Estado de } \\
\text { Mexico }\end{array}$ & Dendroclimatology & TRW \\
\hline 35 & 2014 & Agrociencia & $\begin{array}{l}\text { Correa-Díaz, A.; } \\
\text { Gómez-Guerrero, A.; } \\
\text { Villanueva-Díaz, J.; } \\
\text { Castruita-Esparza, L.; } \\
\text { Martínez-Trinidad, T.; } \\
\text { Cervantes-Martínez, R. }\end{array}$ & $\begin{array}{l}\text { Análisis } \\
\text { dendroclimático de } \\
\text { Ahuehuete (Taxodium } \\
\text { mucronatum Ten.) en el } \\
\text { centro de México. }\end{array}$ & Cupressaceae & $\begin{array}{l}\text { Taxodium mucronatum } \\
\text { Ten. }\end{array}$ & Gallery forest & $\begin{array}{l}\text { Estado de } \\
\text { México, } \\
\text { Querétaro, } \\
\text { Hidalgo and } \\
\text { Morelos }\end{array}$ & Dendroclimatology & TRW \\
\hline 36 & 2014 & $\begin{array}{l}\text { Madera y } \\
\text { bosques }\end{array}$ & $\begin{array}{l}\text { Pompa-García, M.; } \\
\text { Dávalos-Sotelo, R.; } \\
\text { Rodríguez-Téllez, E.; } \\
\text { Aguirre-Calderón, O.; } \\
\text { Treviño-Garza, E. }\end{array}$ & $\begin{array}{l}\text { Sensibilidad climática } \\
\text { de tres versiones } \\
\text { dendrocronológicas } \\
\text { para una conífera } \\
\text { mexicana. }\end{array}$ & Pinaceae & $\begin{array}{l}\text { Pinus cooperi C.E. } \\
\text { Blanco }\end{array}$ & Pine-oak forest & Durango & Dendroclimatology & TRW \\
\hline 37 & 2014 & $\begin{array}{l}\text { International } \\
\text { Journal of } \\
\text { Wildland Fire }\end{array}$ & $\begin{array}{l}\text { Yocom, L.; Fulé, P.; Falk, D.; } \\
\text { García-Domínguez, C.; } \\
\text { Cornejo-Oviedo, E.; } \\
\text { Brown, P.; } \\
\text { Villanueva-Díaz, J.; } \\
\text { Cerano, J.; Montaño, C. }\end{array}$ & $\begin{array}{l}\text { Fine-scale factors } \\
\text { influence fire regimes } \\
\text { in mixed-conifer } \\
\text { forests on three high } \\
\text { mountains in Mexico. }\end{array}$ & Pinaceae & $\begin{array}{l}\text { Pinus hartweegii Lindl., } \\
\text { Pinus strobiformis } \\
\text { Engelm., } \\
\text { Pseudotsuga menziesii } \\
\text { (Mirb.) Franco, } \\
\text { Abies vejarii (Martínez) }\end{array}$ & Pine-oak forest & $\begin{array}{l}\text { Coahuila and } \\
\text { Nuevo León }\end{array}$ & Dendroecology & $\begin{array}{l}\text { TRW, fire } \\
\text { scars }\end{array}$ \\
\hline
\end{tabular}


Table A1. Cont.

\begin{tabular}{|c|c|c|c|c|c|c|c|c|c|c|}
\hline No & Year & Journal & Authors & Title & Family & Tree Species & $\begin{array}{c}\text { Ecosystem } \\
\text { (INEGI } \\
\text { Classification) }\end{array}$ & Region/State & $\begin{array}{c}\text { Area of } \\
\text { Application }\end{array}$ & Variable \\
\hline 38 & 2015 & $\begin{array}{l}\text { Revista } \\
\text { Chapingo Serie } \\
\text { Ciencias } \\
\text { Forestales y del } \\
\text { Ambiente }\end{array}$ & $\begin{array}{l}\text { Chacón-de la Cruz, J; } \\
\text { Pompa-García, M. }\end{array}$ & $\begin{array}{l}\text { Response of tree radial } \\
\text { growth to evaporation, } \\
\text { as indicated by early } \\
\text { and latewood. }\end{array}$ & Pinaceae & $\begin{array}{l}\text { Pinus cooperi C.E. } \\
\text { Blanco }\end{array}$ & Pine forest & Durango & Dendroecology & $\begin{array}{c}\text { EW and } \\
\text { LW }\end{array}$ \\
\hline 39 & 2015 & Atmósfera & $\begin{array}{l}\text { Pompa-García, M.; } \\
\text { Némiga, X. }\end{array}$ & $\begin{array}{l}\text { ENSO index } \\
\text { teleconnection with } \\
\text { seasonal precipitation } \\
\text { in a temperate } \\
\text { ecosystem of northern } \\
\text { Mexico. }\end{array}$ & Pinaceae & $\begin{array}{l}\text { Pinus cooperi C.E. } \\
\text { Blanco }\end{array}$ & Pine-oak forest & Durango & Dendroclimatology & TRW \\
\hline 40 & 2015 & $\begin{array}{l}\text { Madera y } \\
\text { bosques }\end{array}$ & $\begin{array}{l}\text { Carlón-Allende, T.; } \\
\text { Mendoza, M.; } \\
\text { Villanueva-Díaz, J.; } \\
\text { Pérez-Salicrup, D. }\end{array}$ & $\begin{array}{l}\text { Análisis espacial del } \\
\text { paisaje como base para } \\
\text { muestreos } \\
\text { dendrocronológicos: El } \\
\text { caso de la Reserva de } \\
\text { la Biosfera Mariposa } \\
\text { Monarca, México. }\end{array}$ & Pinaceae & $\begin{array}{l}\text { Abies religiosa (Kunth) } \\
\text { Schltdl. \& Cham., } \\
\text { Pinus pseudostrobus } \\
\text { Lindl. }\end{array}$ & $\begin{array}{l}\text { Pine-oak forest } \\
\text { Oyamel forest }\end{array}$ & $\begin{array}{l}\text { Michoacan } \\
\text { and estado de } \\
\text { Mexico }\end{array}$ & Dendroecology & TRW \\
\hline 41 & 2015 & $\begin{array}{l}\text { Madera y } \\
\text { bosques }\end{array}$ & $\begin{array}{l}\text { Villanueva, J.; Cerano, J.; } \\
\text { Olivares, N.; Valles, M.; } \\
\text { Stahle, D.; Cervantes, R. }\end{array}$ & $\begin{array}{l}\text { Respuesta climática del } \\
\text { ciprés (Hesperocyparis } \\
\text { guadalupensis) en Isla } \\
\text { Guadalupe, Baja } \\
\text { California, México. }\end{array}$ & Cupressaceae & $\begin{array}{l}\text { Hesperocyparis } \\
\text { guadalupensis (S. } \\
\text { Watson) Bartel }\end{array}$ & Pine forest & $\begin{array}{c}\text { Baja } \\
\text { California }\end{array}$ & Dendroclimatology & TRW \\
\hline 42 & 2015 & Agrociencia & $\begin{array}{l}\text { Pompa-García, M.; } \\
\text { Camarero, J. }\end{array}$ & $\begin{array}{l}\text { Potencial } \\
\text { dendroclimático de la } \\
\text { madera temprana y } \\
\text { tardía de Pinus cooperi } \\
\text { Blanco. }\end{array}$ & Pinaceae & $\begin{array}{l}\text { Pinus cooperi C.E. } \\
\text { Blanco }\end{array}$ & Pine forest & Durango & Dendroclimatology & EW y LW \\
\hline 43 & 2015 & $\begin{array}{l}\text { International } \\
\text { Journal of } \\
\text { Biometeorology }\end{array}$ & $\begin{array}{l}\text { Pompa-García, M.; } \\
\text { Miranda-Aragón, L.; } \\
\text { Aguirre-Salado, C. }\end{array}$ & $\begin{array}{l}\text { Tree growth response } \\
\text { to ENSO in Durango, } \\
\text { Mexico. }\end{array}$ & Pinaceae & $\begin{array}{l}\text { Pinus cooperi C.E. } \\
\text { Blanco }\end{array}$ & Pine forest & Durango & Dendroecology & TRW \\
\hline 44 & 2015 & $\begin{array}{l}\text { Tree-ring } \\
\text { Research }\end{array}$ & $\begin{array}{l}\text { Pompa-García, M.; } \\
\text { Camarero, J. }\end{array}$ & $\begin{array}{l}\text { Reconstructing } \\
\text { evaporation from pine } \\
\text { tree rings in northern } \\
\text { Mexico }\end{array}$ & Pinaceae & $\begin{array}{l}\text { Pinus cooperi C.E. } \\
\text { Blanco }\end{array}$ & Pine forest & Durango & Dendroclimatology & TRW \\
\hline 45 & 2016 & $\begin{array}{l}\text { Revista } \\
\text { Fitotecnia } \\
\text { Mexicana }\end{array}$ & $\begin{array}{l}\text { Villanueva-Díaz, J.; } \\
\text { Vázquez-Selem, L.; } \\
\text { Gómez-Guerrero, A.; } \\
\text { Cerano-Paredes, J.; } \\
\text { Aguirre-González, N.; } \\
\text { Franco-Ramos, O. }\end{array}$ & $\begin{array}{l}\text { Potencial } \\
\text { dendrocronológico de } \\
\text { Juniperus monticola } \\
\text { Martínez en el Monte } \\
\text { Tláloc, México. }\end{array}$ & Cupressaceae & $\begin{array}{l}\text { Juniperus monticola } \\
\text { Martínez }\end{array}$ & $\begin{array}{l}\text { Coniferous } \\
\text { scrubland }\end{array}$ & $\begin{array}{l}\text { Estado de } \\
\text { Mexico }\end{array}$ & Dendroclimatology & TRW \\
\hline
\end{tabular}


Table A1. Cont.

\begin{tabular}{|c|c|c|c|c|c|c|c|c|c|c|}
\hline No & Year & Journal & Authors & Title & Family & Tree Species & $\begin{array}{c}\text { Ecosystem } \\
\text { (INEGI } \\
\text { Classification) }\end{array}$ & Region/State & $\begin{array}{c}\text { Area of } \\
\text { Application }\end{array}$ & Variable \\
\hline 46 & 2016 & $\begin{array}{l}\text { Madera y } \\
\text { bosques }\end{array}$ & $\begin{array}{l}\text { Díaz-Ramírez, B.; } \\
\text { Villanueva-Díaz, J.; } \\
\text { Cerano-Paredes, J. }\end{array}$ & $\begin{array}{l}\text { Reconstrucción de la } \\
\text { precipitación } \\
\text { estacional con anillos } \\
\text { de crecimiento para la } \\
\text { región hidrológica } \\
\text { Presidio-San Pedro. }\end{array}$ & Pinaceae & $\begin{array}{l}\text { Pinus durangensis } \\
\text { Martínez }\end{array}$ & Pine-oak forest & $\begin{array}{l}\text { Sinaloa and } \\
\text { Nayarit }\end{array}$ & Dendroclimatology & TRW \\
\hline 47 & 2016 & PLoS One & $\begin{array}{l}\text { Pompa-García, M.; } \\
\text { Venegas-González, A. }\end{array}$ & $\begin{array}{l}\text { Temporal Variation of } \\
\text { wood Density and } \\
\text { Carbon in Two } \\
\text { Elevational Sites of } \\
\text { Pinus cooperi in } \\
\text { Relation to Climate } \\
\text { Response in Northern } \\
\text { Mexico. }\end{array}$ & Pinaceae & $\begin{array}{l}\text { Pinus cooperi C.E. } \\
\text { Blanco }\end{array}$ & Pine forest & Durango & Dendroecology & $\begin{array}{c}\text { TRW, wood } \\
\text { density }\end{array}$ \\
\hline 48 & 2016 & Atmósfera & $\begin{array}{l}\text { Pompa-García, M.; } \\
\text { Hadad, M. }\end{array}$ & $\begin{array}{l}\text { Sensitivity of pines in } \\
\text { Mexico to temperature } \\
\text { varies with age. }\end{array}$ & Pinaceae & $\begin{array}{l}\text { Pinus cooperi C.E. } \\
\text { Blanco }\end{array}$ & Pine forest & Durango & Dendroecology & TRW \\
\hline 49 & 2016 & Trees & $\begin{array}{l}\text { González-Cásares, M.; } \\
\text { Pompa-García, M.; } \\
\text { Camarero, J. }\end{array}$ & $\begin{array}{l}\text { Differences in } \\
\text { climate-growth } \\
\text { relationship indicate } \\
\text { diverse drought } \\
\text { tolerances among five } \\
\text { pine species coexisting } \\
\text { in Northwestern } \\
\text { Mexico. }\end{array}$ & Pinaceae & $\begin{array}{l}\text { Pinus lumholtzii B.L. } \\
\text { Rob. \& Fernald, } \\
\text { Pinus durangensis } \\
\text { Martínez, } \\
\text { Pinus arizonica Engelm., } \\
\text { Pinus engelmannii } \\
\text { Carrière, } \\
\text { Pinus leiophylla Schiede } \\
\text { ex Schltdl. \& Cham. }\end{array}$ & Pine-oak forest & Chihuahua & Dendroecology & TRW \\
\hline 50 & 2016 & Trees & $\begin{array}{l}\text { Astudillo-Sánchez, C.; } \\
\text { Villanueva-Díaz, J.; } \\
\text { Endara-Agramont, A.; } \\
\text { Nava-Bernal, G.; } \\
\text { Gómez-Albores, M. }\end{array}$ & $\begin{array}{l}\text { Climatic variability at } \\
\text { the treeline of Monte } \\
\text { Tlaloc, Mexico: a } \\
\text { dendrochronological } \\
\text { approach. }\end{array}$ & Pinaceae & Pinus hartwegii Lindl. & Pine forest & Mexico & Dendroclimatology & TRW \\
\hline 51 & 2016 & $\begin{array}{l}\text { Revista } \\
\text { Chapingo Serie } \\
\text { Ciencias } \\
\text { Forestales y del } \\
\text { Ambiente }\end{array}$ & $\begin{array}{l}\text { Castruita-Esparza; L.; } \\
\text { Correa-Díaz; A.; } \\
\text { Gómez-Guerrero; A.; } \\
\text { Villanueva-Díaz; J.; } \\
\text { Ramírez-Guzmán, M.; } \\
\text { Velázquez-Martínez, A.; } \\
\text { Ángeles-Pérez, G. }\end{array}$ & $\begin{array}{l}\text { Basal area increment } \\
\text { series of dominant } \\
\text { trees of } \\
\text { Pseudotsuga menziesii } \\
\text { (Mirb.) Franco show } \\
\text { periodicity according } \\
\text { to global climate } \\
\text { patterns. }\end{array}$ & Pinaceae & $\begin{array}{l}\text { Pseudotsuga menziesii } \\
\text { (Mirb.) Franco }\end{array}$ & Pine-oak forest & Chihuahua & Dendroecology & $\begin{array}{l}\text { TRW, basal } \\
\text { area } \\
\text { increment }\end{array}$ \\
\hline
\end{tabular}


Table A1. Cont.

\begin{tabular}{|c|c|c|c|c|c|c|c|c|c|c|}
\hline No & Year & Journal & Authors & Title & Family & Tree Species & $\begin{array}{c}\text { Ecosystem } \\
\text { (INEGI } \\
\text { Classification) }\end{array}$ & Region/State & $\begin{array}{c}\text { Area of } \\
\text { Application }\end{array}$ & Variable \\
\hline 52 & 2016 & $\begin{array}{l}\text { Botanical } \\
\text { Sciences }\end{array}$ & $\begin{array}{l}\text { Ortiz-Quijano, A.; } \\
\text { Sánchez-González, A.; } \\
\text { López-Mata, L.; } \\
\text { Villanueva-Díaz, J. }\end{array}$ & $\begin{array}{l}\text { Population structure of } \\
\text { Fagus grandifolia subsp. } \\
\text { Mexicana in the cloud } \\
\text { forest of Hidalgo state, } \\
\text { Mexico. }\end{array}$ & Fagaceae & $\begin{array}{l}\text { Fagus grandifolia subsp. } \\
\text { mexicana (Martínez) } \\
\text { A.E. Murray }\end{array}$ & $\begin{array}{l}\text { Mountain } \\
\text { mesophilous } \\
\text { forest }\end{array}$ & Hidalgo & Dendroecology & TRW \\
\hline 53 & 2016 & $\begin{array}{l}\text { Tree-ring } \\
\text { Research }\end{array}$ & $\begin{array}{l}\text { Torbenson, M.; Stahle, D.; } \\
\text { Villanueva-Díaz, J.; } \\
\text { Cook, E.; Griffin, D. }\end{array}$ & $\begin{array}{l}\text { The relationship } \\
\text { between earlywood } \\
\text { and latewood } \\
\text { ring-Growth across } \\
\text { North America. }\end{array}$ & $\begin{array}{l}\text { Pinaceae, } \\
\text { Fagaceae, } \\
\text { Cupressaceae }\end{array}$ & $\begin{array}{l}\text { Pseudotsuga menziesii } \\
\text { (Mirb.) Franco, } \\
\text { Pinus engelmannii } \\
\text { Carrière }\end{array}$ & & - & Dendroecology & $\begin{array}{l}\text { EW and } \\
\text { LW }\end{array}$ \\
\hline 54 & 2016 & Dendrochronologia & $\begin{array}{l}\text { Carlón, T.; Mendoza, M.; } \\
\text { Pérez-Salicrup, D.; } \\
\text { Villanueva-Díaz, J.; Lara, A. }\end{array}$ & $\begin{array}{l}\text { Climatic responses of } \\
\text { Pinus pseudostrobus and } \\
\text { Abies religiosa in the } \\
\text { Monarch Butterfly } \\
\text { Biosphere Reserve, } \\
\text { Central Mexico. }\end{array}$ & Pinaceae & $\begin{array}{l}\text { Pinus pseudostrobus } \\
\text { (Lindl), Abies religiosa } \\
\text { (Kunth) Schltdl. \& } \\
\text { Cham. }\end{array}$ & Pine forest & $\begin{array}{l}\text { Michoacan } \\
\text { and estado de } \\
\text { Mexico }\end{array}$ & Dendroecology & TRW \\
\hline 55 & 2016 & $\begin{array}{l}\text { Environmental } \\
\text { Geochemistry } \\
\text { and Health }\end{array}$ & $\begin{array}{l}\text { Morton-Bermea, O.; } \\
\text { Beramendi-Orosco, L.; } \\
\text { Martínez-Reyes, Á.; } \\
\text { Hernández-Âlvarez, E.; } \\
\text { González-Hernández, G. }\end{array}$ & $\begin{array}{l}\text { Increase in platinum } \\
\text { group elements in } \\
\text { Mexico City as } \\
\text { revealed from growth } \\
\text { rings of Taxodium } \\
\text { mucronatum ten. }\end{array}$ & Cupressaceae & $\begin{array}{l}\text { Taxodium mucronatum } \\
\text { Ten. }\end{array}$ & Gallery forest & $\begin{array}{c}\text { Cd. de } \\
\text { Mexico DF }\end{array}$ & Dendroecology & $\begin{array}{l}\text { TRW, } \\
\text { chemical } \\
\text { elements }\end{array}$ \\
\hline
\end{tabular}

Variables' abbreviations: TRW, tree-ring width; EW, earlywood width; LW, latewood width. 


\section{References}

1. Fritts, H.C. Tree-Rings and Climate; Academia Press: London, UK, 1976; p. 567.

2. Pompa-García, M.; Hadad, M.A. Sensitivity of pines in Mexico to temperature varies with age. Atmósfera 2016, 29, 209-219. [CrossRef]

3. Arreola-Ortiz, M.R.; Návar-Cháidez, J.D.J. Análisis de sequías y productividad con cronologías de Pseudotsuga menziesii Rob. \& Fern., y su asociación con El Niño en el nordeste de México. Investig. Geogr. 2010, 71, 7-20.

4. Correa-Díaz, A.; Gómez-Guerrero, A.; Villanueva-Díaz, J.; Castruita-Esparza, L.U.; Martínez-Trinidad, T.; Cervantes-Martínez, R. Análisis dendroclimático de ahuehuete (Taxodium mucronatum Ten.) en el centro de México. Agrociencia 2014, 48, 537-551.

5. Chhin, S. Influence of climate on the growth of hybrid poplar in Michigan. Forests 2010, 1, 209-229. [CrossRef]

6. Douglass, A.E. A method of estimating rainfall by the growth of trees. Bull. Am. Geogr. Soc. 1914, 46, 321-335. [CrossRef]

7. Worbes, M. One hundred years of tree-ring research in the tropics-A brief history and an outlook to future challenges. Dendrochronologia 2002, 20, 217-231. [CrossRef]

8. Tschinkel, H.M. Anillos de crecimiento anual en Cordia alliodora. Turrialba 1966, 16, 73-80.

9. Mariaux, A. Les cernes dans les bois tropicaux africains. Nature et Périodicité. Revue Bois et Forêts Des Tropiques 1967, 114, 23-37.

10. Boninsegna, J.A.; Villalba, R.; Amarilla, L.; Ocampo, J. Studies on tree rings, growth rates and age-size relationships of tropical tree species in Misiones, Argentina. IAWA Bull. 1989, 10, 161-169. [CrossRef]

11. Schulman, E. Dendrochronology in Mexico, I. Tree-Ring Bull. 1944, 10, 18-24.

12. Hughes, M.K. Dendrochronology in climatology-The state of the art. Dendrochronologia 2002, 20, 95-116. [CrossRef]

13. Scott, S.D. Dendrochronology in Mexico; Papers of the Laboratory of Tree-Ring Research; University of Arizona Press: Tucson, AZ, USA, 1966; p. 80.

14. Villanueva-Díaz, J.; Cerano, J.; Stahle, D.W.; Therrell, M.D.; Vázquez, L.; Morán, R.; Luckman, B.H. Árboles Viejos del Centro-Norte de México: Importancia Ecológica y Paleoclimática; Folleto Científico 20; INIFAP-CENID-RASPA: Gómez Palacio, Durango, Mexico, 2006; p. 46.

15. Villanueva-Díaz, J.; Vázquez-Selem, L.; Gómez-Guerrero, A.; Cerano-Paredes, J.; Aguirre-González, N.A.; Franco-Ramos, O. Potencial dendrocronológico de Juniperus monticola Martínez en el monte Tláloc, México. Rev. Fitotec. Mex. 2016, 39, 175-185.

16. Tomazello, M.; Roig, F.A.; Zevallos, P.A. Dendrocronología y dendroecología tropical: Marco histórico y experiencias exitosas en los países de América Latina. Ecol. Boliv. 2009, 44, 73-82.

17. Astudillo-Sánchez, C.C.; Villanueva-Díaz, J.; Endara-Agramont, A.R.; Nava-Bernal, G.E.; Gómez-Albores, M.A. Climatic variability at the treeline of Monte Tlaloc, Mexico: A dendrochronological approach. Trees 2017, 31, 441-453. [CrossRef]

18. Díaz-Ramírez, B.; Villanueva-Díaz, J.; Cerano-Paredes, J. Reconstrucción de la precipitación estacional con anillos de crecimiento para la región hidrológica Presidio-San Pedro. Madera Bosques 2016, 22, 111-123. [CrossRef]

19. Stahle, D.W.; Cook, E.R.; Burnette, D.J.; Villanueva, J.; Cerano, J.; Burns, J.N.; Griffin, D.; Cook, B.I.; Acuña, R.; Torbenson, M.C.A.; et al. The Mexican Drought Atlas: Tree-ring reconstructions of the soil moisture balance during the late pre-Hispanic, colonial, and modern eras. Quat. Sci. Rev. 2016, 149, 34-60. [CrossRef]

20. Brienen, R.J.W.; Schöngart, J.; Zuidema, P.A. Tree rings in the tropics: Insights into the ecology and climate sensitivity of tropical trees. Trop. Tree Physiol. 2016, 6, 439-461.

21. Mendivelso, H.A.; Camarero, J.J.; Gutiérrez, E. Dendrocronología en bosques neotropicales secos: Métodos, avances y aplicaciones. Ecosistemas 2016, 25, 66-75. [CrossRef]

22. Perry, J.P. The pines of Mexico and Central America; Timber Press: Portland, OR, USA, 1991; p. 231.

23. González-Elizondo, M.; Jurado, E.; Návar, J.; GonzálezElizondo, M.S.; Villanueva, J.; Aguirre, O.; Jiménez, J. Tree-rings and climate relationships for Douglas-fir chronologies from the Sierra Madre Occidental, Mexico: A 1681-2001 rain reconstruction. For. Ecol. Manag. 2005, 213, 39-53. [CrossRef] 
24. Villanueva-Díaz, J.; Stahle, D.W.; Luckman, B.H.; Cerano-Paredes, J.; Therrell, M.D.; Cleaveland, M.K. Winter-spring precipitation reconstructions from tree rings for northeast Mexico. Clim. Chang. 2007, 83, 117-131. [CrossRef]

25. Griesbauer, H.; Scott, G.D. Assessing the climatic sensitivity of Douglas-fir at its northern range margins in British Columbia, Canada. Trees 2010, 24, 375-389. [CrossRef]

26. Torbenson, M.C.A.; Stahle, D.W.; Villanueva, J.; Cook, E.R.; Griffin, D. The relationship between earlywood and latewood ring-growth across North America. Tree-Ring Res. 2016, 72, 53-66. [CrossRef]

27. Pompa-García, M.; Cerano-Paredes, J.; Fulé, P.Z. Variation in radial growth of Pinus cooperi in response to climatic signals across an elevational gradient. Dendrochronologia 2013, 31, 198-204. [CrossRef]

28. Secretaria de Medio Ambiente y Recursos Naturales (SEMARNAT). Norma Oficial Mexicana NOM.059-SEMARNAT; Diario Oficial de la Federación 30 de diciembre de 2010; SEMARNAT: Mexico City, Mexico, 2010.

29. López-Upton, J.; Valdez-Lazalde, J.R.; Ventura-Ríos, A.; Vargas-Hernández, J.J.; Guerra-de-la-Cruz, V. Extinction risk of Pseudotsuga menziesii populations in the central region of Mexico: An AHP analysis. Forests 2015, 6, 1598-1612. [CrossRef]

30. Jiménez, J.A.G. Dendrocronología en el trópico: Aplicaciones actuales y potenciales. Colomb. For. 2011, 14, 97-111. [CrossRef]

31. Gutiérrez, L.A.B.; Ramos, G.M.V. Anatomía de anillos de crecimiento de 80 especies arbóreas potenciales para estudios dendrocronológicos en la Selva Central, Perú. Rev. Biol. Trop. 2013, 61, 1025-1037.

32. Pompa-García, M.; Rodríguez-Flores, F.J.; Aguirre-Salado, C.A.; Miranda-Aragón, L. Influencia de la evaporación en el crecimiento forestal. Agrociencia 2013, 47, 829-836.

33. Pompa-García, M.; Jurado, E. Seasonal precipitation reconstruction and teleconnections with ENSO based on tree ring analysis of Pinus cooperi. Theor. Appl. Climatol. 2014, 117, 495-500. [CrossRef]

34. Díaz, S.C.; Therrell, M.D.; Stahle, D.W.; Cleaveland, M.K. Chihuahua (Mexico) winter-spring precipitation reconstructed from tree-rings, 1647-1992. Clim. Res. 2002, 22, 237-244. [CrossRef]

35. Sheppard, P.R.; Ort, M.H.; Anderson, K.C.; Elson, M.D.; Vázquez-Selem, L.; Clemens, A.W.; Little, N.C.; Speakman, R.J. Multiple dendrochronological signals indicate the eruption of Paricutin volcano, Michoacan, Mexico. Tree-Ring Res. 2008, 64, 97-108. [CrossRef]

36. González-Cásares, M.; Pompa-García, M.; Camarero, J.J. Differences in climate-growth relationship indicate diverse drought tolerances among five pine species coexisting in Northwestern Mexico. Trees 2016, 31, 531-544. [CrossRef]

37. Cleaveland, M.K.; Cook, E.R.; Stahle, D.W. Secular variability of the ENSO signal detected in tree-ring data from Mexico and the southern United States. In El Nino: Historical and Paleoclimatic Aspects of the Southern Oscillation; Diaz, H., Markgraf, V., Eds.; Cambridge University Press: Cambridge, NY, USA, 1992; pp. 271-291.

38. Stahle, D.W.; Cleaveland, M.K. Southern Oscillation extremes reconstructed from tree rings of the Sierra Madre Occidental and southern Great Plains. J. Clim. 1993, 6, 129-140. [CrossRef]

(C) 2017 by the authors. Licensee MDPI, Basel, Switzerland. This article is an open access article distributed under the terms and conditions of the Creative Commons Attribution (CC BY) license (http:/ / creativecommons.org/licenses/by/4.0/). 